\title{
34 Appendizitis
}

Stefan Farke

\subsection{Kapitelzusammenfassung}

Die akute Appendizitis ist die häufigste Ursache akuter Unterbauchschmerzen. Bei der Diagnostik stehen Anamnese, klinische Untersuchung, Bestimmung von Laborparametern und Sonographie im Vordergrund. Abhängig von der Schwere der Entzündung wird nach der Diagnostik entschieden, ob eine akute chirurgische Intervention notwendig ist. Für die Operation ist der minimal-invasive Zugang heute der Goldstandard. Differentialdiagnosen der Appendizitis sind andere entzündliche Darmerkrankungen, Neoplasien oder Erkrankungen des inneren Genitales bei Frauen. Bei unkomplizierter Appendizitis kann eine konservativ-antibiotische Behandlung erfolgreich sein.

\subsection{Definition}

Unter einer Appendizitis versteht man die Entzündung des Wurmfortsatzes. Der Wurmfortsatz (Appendix vermiformis) findet sich am Beginn des Dickdarms als Anhängsel am Blinddarm (Coecal-Pol) Umgangssprachlich spricht man auch oft von einer Blinddarm-Entzündung, was allerdings anatomisch nicht korrekt ist, da nicht der Blinddarm, sondern der daran „anhängende“ Wurmfortsatz entzündet ist.

\section{3 Åtiologie}

Die häufigste Ursache einer Appendizitis ist wahrscheinlich eine Obstruktion des Lumens durch Koprolithen oder Fremdkörper, weniger häufige Ursachen sind Neubildungen oder Parasiten. Auch andere Infektionen können eine Appendizitis verursachen, z. B. gastrointestinale Infektionen oder Infekte der Atemwege. Solche Begleitinfekte sollten bei der Erhebung der Anamnese erfragt werden.

\subsection{Pathogenese}

Zur Pathogenese der Appendizitis wird im Allgemeinen eine Entwicklung in verschiedenen Stadien zunehmender Schwere beschrieben.

- Initialstadium der Appendizitis ist die katarrhalische Entzündung, die mit einer Rötung und leichten Schwellung der Appendix einhergeht und prinzipiell reversibel ist. 
- Phlegmonöse oder ulcero-phlegmonöse Entzündungen sind gekennzeichnet durch eine erhebliche Schwellung und Rötung, oft mit Auflagerungen von Eiter (Abb. 34.1).

- Gangränöse Appendizitiden zeichnen sich zusätzlich zum Bild der phlegmonösen Entzündung durch eine oder mehrere gangränös/nekrotische Stellen an der Appendix aus. Meist besteht auch eine zumindest lokale Peritonitis (Abb.34.2).

- Die perforierte Appendizitis ist die am weitesten fortgeschrittene Entzündung.

Unklar ist, ob alle Appendizitiden diese Stadien durchlaufen, nach der klinischen Erfahrung gibt es vor allem zeitlich sehr unterschiedliche Verläufe, unter anderem auch sehr kurze Anamnesen vor einer Perforation mit Peritonitis.
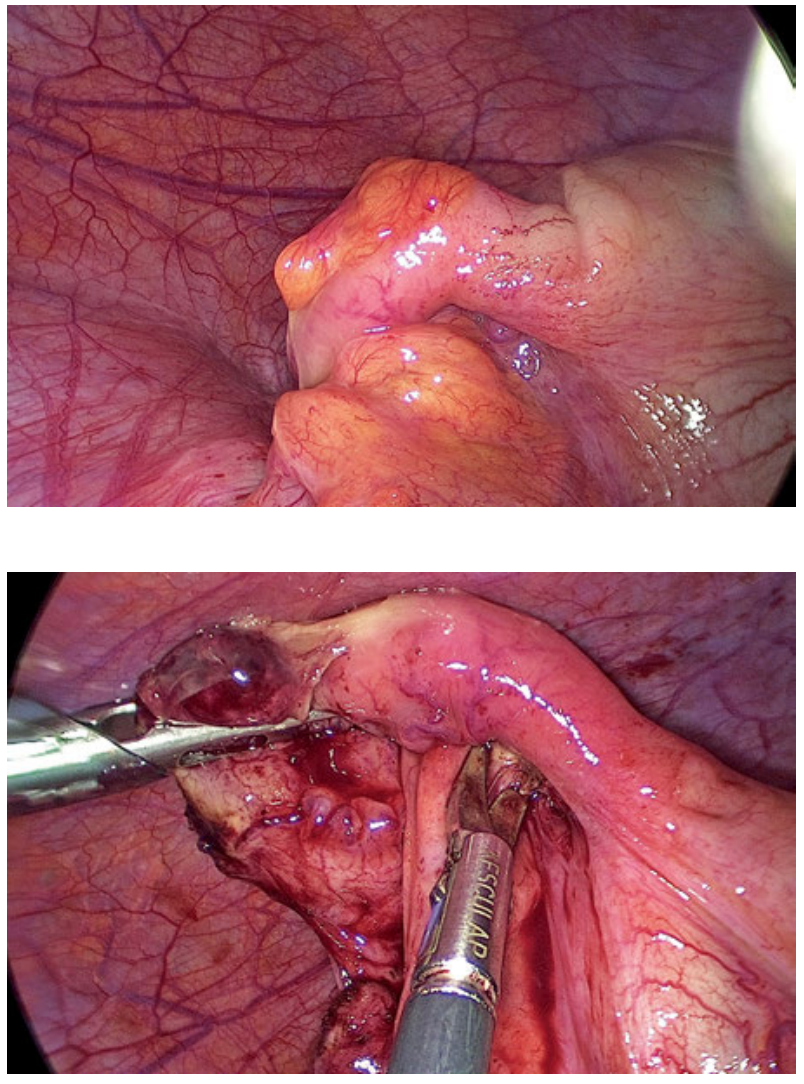

Abb.34.1: Phlegmonöse Appendizitis.

Abb. 34.2: Gangränöse Appendizitis. 


\subsection{Inzidenz/Epidemiologie}

Zur Häufigkeit der Appendizitis haben Ferris et al. aktuell Zahlen eines systematischen Reviews aus einer Vielzahl von Studien zur Inzidenz der Appendizitis publiziert. Danach liegt die Inzidenz im 21. Jahrhundert etwa um 100-150 pro 100.000 Einwohner mit deutlichen regionalen Unterschieden. Während sie in Nord-Amerika bei 100/100.000 Einwohnern liegt, reichen die Raten in Europa von 105/100.000 in OstEuropa bis 151/100.000 Einwohnern in West-Europa. Dabei nimmt die Inzidenz der Appendektomie seit 1990 ab, während die Inzidenz der Appendizitis stabil bleibt. Auffällig in den publizierten Zahlen ist die hohe Inzidenz der Appendizitis oder -ektomie in „Neu-Industrialisierten“ Ländern in Asien (Korea 206/100.000), im mittleren Osten (Türkei 160/100.000) oder Süd-Amerika (Chile 202/100.000).

Der Appendizitis tritt am häufigsten im Alter zwischen 10 und 30 Jahren auf, sie gilt allgemein als die häufigste Ursache für ein akutes Abdomen und das geschätzte Lebenszeit-Risiko an einer Appendizitis zu erkranken beträgt ca. $8 \%$.

\subsection{Klassifikation}

Eine allgemein akzeptierte und validierte Klassifikation der Appendizitis existiert bisher nicht. Im klinischen Alltag wird meist zwischen einer akuten und einer nichtakuten (oder sonstigen) Appendizitis unterschieden. Dies entspricht auch den Abbildungsmöglichkeiten der Erkrankung in der ICD Klassifikation. Hier werden unter Erkrankungen der Appendix vier Diagnose-Optionen aufgeführt:

- K35 akute Appendizitis

- K36 sonstige Appendizitis

- K37 nicht näher bezeichnete Appendizitis

- K38 sonstige Krankheiten der Appendix

In der präoperativen Evaluation des Krankheitsbildes ist vor allem die Unterscheidung in akut im Sinne von operationspflichtig und nicht akut wichtig. Diese Einteilung entspricht im Wesentlichen der Differenzierung in kompliziert und nicht-kompliziert.

Intraoperativ wird die Appendizitis nach dem makroskopischen Bild eingeteilt:

- blande Appendix ohne entzündliche Veränderungen

- Gefäß-injizierte Appendix (leichte Entzündung, katarrhalische Entzündung)

- phlegmonöse Entzündung (Abb. 34.1)

- gangränöse Entzündung (Abb. 34.2)

- perforierte Appendizitis 


\subsection{Symptomatik}

Die führende Symptomatik ist der Schmerz im rechten Unterbauch. Dabei beginnt bei „lehrbuchmäßigem“ Verlauf der Schmerz am Nabel und wandert im Verlauf in den rechten Unterbauch. Bei der klassischen Symptomatik besteht bei der akuten Appendizitis ein deutlicher Druckschmerz im rechten Unterbauch, Peritonismus-Zeichen mit Klopfschmerz, Loslassschmerz (auch kontralateral) und Abwehrspannung weisen auf eine Operationspflichtigkeit hin.

Diese „klassischen“ Beschwerden einer Appendizitis sind in vielen Fällen nicht oder nur teilweise vorhanden. Insbesondere bei atypischer Lage der Appendix (z. B. retrocoecal, subhepatisch) können die Symptome variieren. Daher ist bei der Untersuchung vor allem die Festlegung wichtig, ob eine akute OP Indikation besteht oder nicht. Die tatsächliche Ursache der Unterbauchschmerzen kann dann im Rahmen der diagnostischen Laparoskopie ermittelt werden.

\subsection{Diagnostik}

Bei der Diagnostik der Appendizitis steht die klinische Untersuchung mit Anamnese, körperlicher Untersuchung und Laboruntersuchungen im Vordergrund. Daneben stellt die Sonographie des Abdomens eine jederzeit verfügbare, nicht invasive und kostengünstige Untersuchungsmethode dar, mit der insbesondere in nicht eindeutigen Fällen eine Entscheidung zum Procedere herbeigeführt werden kann. In der Akutsituation steht zunächst die Entscheidung im Vordergrund, ob eine akute Operationsindikation besteht oder nicht. Dies ist im Wesentlichen vom klinischen Bild abhängig.

Laboruntersuchungen gehören zur Diagnostik der Appendizitis, die Interpretation der Werte in Bezug auf ihre Aussagekraft zur Bestätigung einer Appendizitis kann schwierig sein. Farooqui und Mitarbeiter haben dazu ihr Ergebnis uni- und multivariater Analysen publiziert, danach ist eine Kombination verschiedener Marker am aussagekräftigsten und eine Erhöhung des CRP, der Leukozyten, des Bilirubins und der Alanin-Aminotransferase (ALT) sprechen bei Schmerzen im rechten Unterbauch für eine akute oder perforierte Appendizitis.

Wenn der abdominelle Befund eine akute Operationsnotwendigkeit bedingt, ist keine weitere Diagnostik indiziert. Wenn nicht akut operiert werden muss, kann neben der Sonographie eine weitere Diagnostik angezeigt sein. Dazu gehören dann abhängig von der Symptomatik und der Anamnese eine Computertomographie des Abdomens oder eine Endoskopie. In einer aktuellen Metaanalyse zur Computer-Tomographie des Abdomens bei Patienten mit akuten Schmerzen im rechten Unterbauch kommen Krajewski et al. zu dem Ergebnis, das durch eine CT Untersuchung die Rate der negativen Appendektomien deutlich gesenkt werden kann, ohne dass durch die Verzögerung der Behandlung die Perforationsrate steigt. Allerdings führt diese vor 
allem in den Vereinigten Staaten geübte Praxis zu einer erheblichen Zunahme der Strahlenexposition, so dass die CT-Diagnostik in Europa deutlich weniger eingesetzt wird.

\subsection{Differentialdiagnosen}

Eine große Zahl verschiedener pathologischer Veränderungen, die Schmerzen im Unterbauch verursachen, kommt als Differentialdiagnosen der Appendizitis in Frage:

Erkrankungen des inneren Genitale: insbesondere bei jungen Frauen stellen v. a. entzündliche Erkrankungen eine Differentialdiagnose der Appendizitis dar. Eine Schwangerschaft muss ausgeschlossen werden.

Entzündliche Darmerkrankungen: alle entzündlichen Darmerkrankungen stellen wichtige Differentialdiagnosen dar, insbesondere die Erstmanifestation eines Morbus Crohn ist im Akutfall präoperativ schwer von einer Appendizitis zu differenzieren. Auch eine Divertikulitis des Kolon ascendens kann gleichartige Symptome wie eine typische Appendizitis verursachen.

Neoplasien: Insbesondere bei älteren Patientinnen und Patienten muss bei Schmerzen im rechten Unterbauch ein Kolonkarzinom als Ursache in Betracht gezogen werden.

\subsection{Therapie konservativ}

Die konservative Therapie der Appendizitis wird in den letzten Jahren besonders im angloamerikanischen Sprachraum intensiv diskutiert. In einer aktuellen prospektiven Studie wurde die konservativ antibiotische Behandlung von Patienten mit als unkompliziert eingeschätzter Appendizitis nach initialer Computertomographie untersucht. Bei diesem Ansatz versagte die konservative Therapie in 2,5\% der Fälle, bei denen während des initialen Krankenhausaufenthaltes eine Appendektomie notwendig war. Von den initial erfolgreich konservativ Behandelten mussten im Jahr nach der Behandlung 12,2\% sekundär appendektomiert werden. In Anbetracht der aktuellen Daten scheint eine konservativ-antibiotische Behandlung einer unkomplizierten Appendizitis mit guten Erfolgsaussichten möglich, allerdings ist die sichere Differenzierung einer unkomplizierten Appendizitis wohl nur durch eine Computertomographie des Abdomens möglich. 


\subsection{Therapie operativ}

Die operative Entfernung der Appendix stellt den Goldstandard der Therapie der akuten Appendizitis dar. Heute ist dabei die laparoskopische Operation das Standardverfahren. Nach der Einführung der minimal invasiven Operation in die Therapie der Appendizitis wurde der Zugangsweg in einer Vielzahl von Publikationen untersucht. Aktuell summiert eine Analyse aller zwischen 2010 und 2016 publizierten prospektivrandomisierten Studien und nationalen Register-Kohorten Studien die Ergebnisse von 25 Studien. Dabei beträgt der Anteil der minimal invasiven Operationen 86\%. Die offene Appendektomie bleibt eine sichere und effektive Technik. Single-Port laparoskopische Appendektomie und NOTES Techniken sind bisher nicht sehr verbreitet. Zusammenfassend sehen die Autoren den laparoskopischen Zugang als „State of the Art“.

Laparoskopische Operation: Die Operation erfolgt in Allgemeinanästhesie in Rückenlage. Meist werden drei Trokare verwendet, neben einem Kameratrokar (je nach Optik 5 bzw. $10 \mathrm{~mm}$ ) ein Arbeitstrokar mit $12 \mathrm{~mm}$ und einer mit $5 \mathrm{~mm}$ Durchmesser. Dabei wird der Kameratrokar am Nabel, der große Arbeitstrokar im lateralen linken Unterbauch auf Höhe der Spina iliaka anterior superior und der zweite Arbeitstrokar im mittleren oder im rechten Unterbauch platziert. Für den ersten Trokar, meist der Kameratrokar am Nabel, wird im eigenen Vorgehen ein offener Zugang bevorzugt, bei Verwendung kleiner Optiken $(5 \mathrm{~mm} / 3 \mathrm{~mm}$ ) auch ein optischer Zugangstrokar. Nach Aufbau des Capnoperitoneums erfolgt eine diagnostische Laparoskopie, bei der das gesamte Abdomen inspiziert wird. Nach der Bestätigung der Appendizitis-Diagnose wird die Appendix exponiert, ggf. durch Inzision oder stumpfes Lösen von Adhäsionen und Fixierungen mobilisiert und inspiziert. Zur Appendektomie sind eine Dissektion des Mesenteriolums mit sicherer Versorgung der Arteria appendicularis und ein Absetzen der Appendix an der Basis unmittelbar oberhalb des Coecalpols nötig. Im eigenen Vorgehen wird eine Durchtrennung der Arterie zwischen Clips bevorzugt, die Versorgung des Appendixstumpfes erfolgt bei entzündungsfreier oder mäßig entzündeter Appendixbasis mit Kunststoff-Clips (Abb. 34.3), bei stärkeren entzündlichen Veränderung mit einem Klammernaht-Gerät (Abb. 34.4).

Für die Bergung der abgetragenen Appendix sollte großzügig der Einsatz eines Bergebeutels gewählt werden, wenn eine Bergung durch den größten Trokar nicht problemlos möglich erscheint. Wenn der Befund der Appendix die präoperativen Beschwerden nicht sicher erklärt, müssen andere Ursachen für die Symptomatik ausgeschlossen werden. Dazu gehört das Durchmustern des Dünndarms zum Ausschluss eines Meckel'schen Divertikels, das bei Erwachsenen etwa 60-90 cm vor der Bauhin`schen Klappe zu erwarten ist und zusätzlich bei Frauen die Inspektion des inneren Genitales. Die Operation wird nach dem Bergen des Präparates mit dem Entfernen der Trokare unter Sicht und dem Ablassen des Capnoperitoneums beendet. 

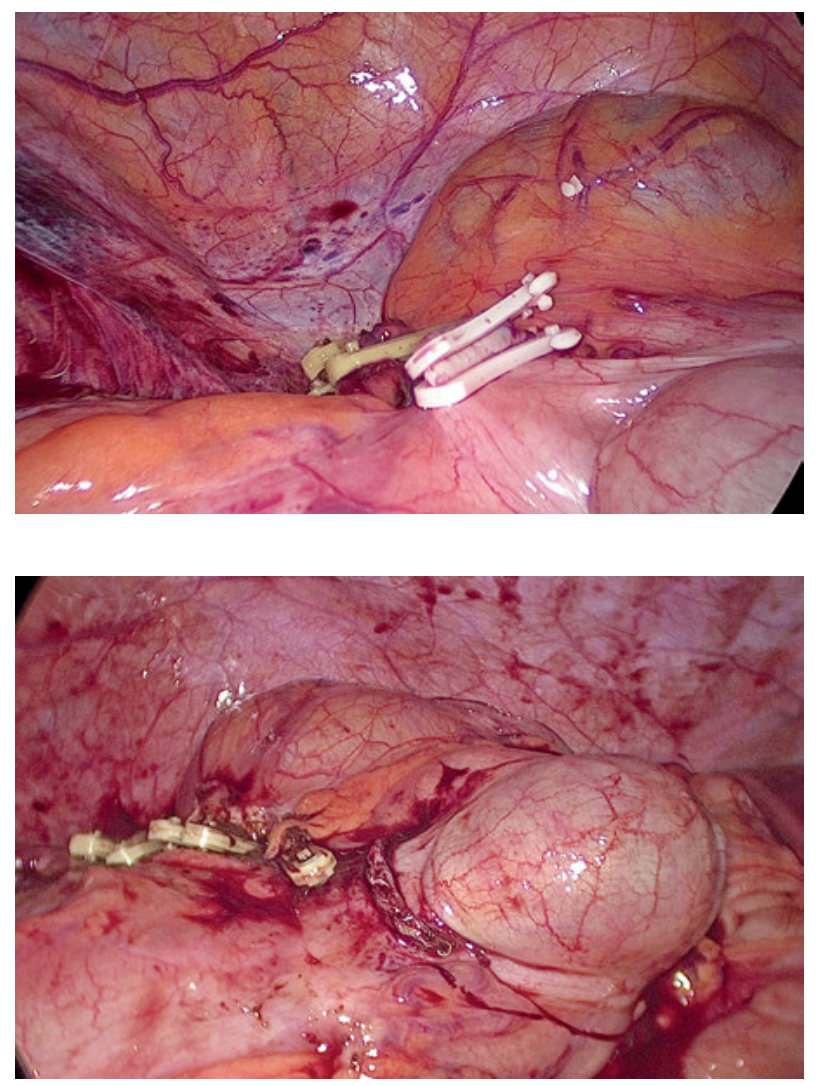

Abb. 34.3: Appendixstumpf mit Kunststoff-Clips.

Abb. 34.4: Appendixstumpf mit Klammern.

Im eigenen Vorgehen werden Drainagen nur bei perforierter Appendizitis mit eitriger Peritonitis eingebracht, bevorzugt Easy Flow-Drainagen.

Konventionelle offene Operation: Indikation zur offenen Operation ist heute vor allem die Konversion bei laparoskopisch nicht beherrschbarem Situs. Daher erfolgt die Operation meist durch eine (Unterbauch-) Medianlaparotomie. Nach Einstellen des Situs wird auch bei der offenen Operation die Appendix exponiert und dann das Mesenteriolum zwischen Overholtklemmen disseziert. Danach wird die Appendix an der Basis ligiert und über der Ligatur abgesetzt. Zur Sicherung des Stumpfes kann dieser nach Anlegen einer Tabaksbeutelnaht um die Appendixbasis in das Coecum eingestülpt werden oder mit einer seromuskulären Z-Naht versenkt werden. 


\subsection{Komplikationen}

Komplikationen der Appendizitis: Die Appendizitis kann bei ausbleibender oder unzureichender Behandlung fortschreiten. Durch Perforation und Ausbreitung der Infektion im Bauchraum kann eine schwere septische Peritonitis entstehen.

Komplikationen der Operation: Intraoperative Komplikationen der Appendektomie sind am häufigsten Blutungen oder Verletzungen von Nachbarorganen. Bei laparoskopischem Vorgehen muss auf die mögliche Konversion auf eine offene Operation hingewiesen werden, die Konversion ist in diesem Sinne aber keine Komplikation. Zu den wichtigen postoperativen Komplikationen gehören die Insuffizienz des Appendixstumpfes mit Peritonitis; Nachblutungen, mechanischer oder paralytischer Ileus oder die Entwicklung eines Platzbauches. Auch Wundinfektionen, Wundheilungsstörungen oder andere allgemein-postoperative Komplikationen sind möglich (Thrombose, Embolie, Pneumonie). Die häufigsten Komplikationen nach Appendektomie sind minor-Komplikationen wie Wundheilungsstörungen oder -infektionen.

\subsection{Besonderheiten}

Appendektomie bei makroskopisch blander Appendix aber typischer Klinik: Im Zeitalter der offenen Appendektomie war es Standard, auch bei intraoperativ unauffälliger Appendix diese zu entfernen, da der typische Schnitt die Erwartung bedingte, dass die Appendix bereits entfernt wurde. Nach der Einführung der laparoskopischen Technik wurde von dieser Praxis abgewichen. Heute ist das Vorgehen in dieser Frage nicht einheitlich. Im eigenen Handeln wird bei einer Operation unter Verdacht auf Appendizitis die Appendix auch entfernt, nur bei einem eindeutig die Beschwerden erklärenden anderen Befund und völlig blander Appendix kann die Appendix belassen werden.

\subsection{Prävention}

Eine nachgewiesen effektive Prävention zur Vermeidung einer Appendizitis existiert nicht. 


\section{Weiterführende Literatur}

Al-Mulhim AS. Readmission after antibiotic management of uncomplicated acute appendicitis in adults: prospective study. Eur J Trauma Emerg Surg. 2018; Oct 26. doi: 10.1007/s00068-0181038-0. [Epub ahead of print].

Bhangu A, et al. Acute appendicitis: modern understanding of pathogenesis, diagnosis, and management. Lancet. 2015;386(10000):1278-1287.

Farooqui W. The diagnostic value of a panel of serological markers in acute appendicitis. Scand J Surg. 2015;104(2):72-78.

Ferris M, et al. The Global Incidence of Appendicitis: A Systematic Review of Population-based Studies. Ann Surg. 2017;266(2):237-241.

Hoffmann M, Anthuber M. [Rational diagnostics of acute appendicitis]. Chirurg. 2019;90(3):173-177. doi: 10.1007/s00104-018-0755-6.

Krajewski S, et al. Impact of computed tomography of the abdomen on clinical outcomes in patients with acute right lower quadrant pain: a meta-analysis. J can chir. 2011;54(1).

Kujath C, Kollmar O, Ghadimi BM. [Is acute appendicitis a surgical emergency?] Chirurg. 2019;90(3):183-185. doi: 10.1007/s00104-018-0757-4.

Sauvain MO, et al. Delaying surgery to perform CT scans for suspected appendicitis decreases the rate of negative appendectomies without increasing the rate of perforation nor postoperative complications. Langenbecks Arch Surg. 2016;401(5):643-649.

Sohn M, et al. Surgical management of acute appendicitis in adults: A review of current techniques. Int J Surg. 2017;48:232-239. 
